\section{Pflegesymposium in Bochum Ein Rückblick auf das Symposium 2008}

Am 20. November 2008 fand in Bochum das 12. Pflegesymposium des Arbeitskreises Transplantationspflege (AKTX) statt. Die Themen der Vorträge und der vorgestellten Poster waren sehr vielfältig und informativ. Die 139 teilnehmenden Pflegenden tauschten sich über neue Entwicklungen in der Transplantationspflege aus und nahmen viel Wissen mit, das sie in der täglichen Arbeit umsetzen können.

Der Kostendruck auf die Krankenhäuser wächst, weshalb umfangreiche Umgestaltungen wie Fusionen und Personalkürzungen notwendig sind. Daher ist es umso wichtiger, dass sich die Pflege weiter organisiert und ihre Arbeit, ihr Wissen, ihre Erfahrungen und Kenntnisse einer breiten Öffentlichkeit präsentiert. Eine gute Plattform dafür bietet das jährlich stattfindende Pflegesymposium des AKTX.

\section{Pflegesymposien stärken die Kompetenzen der Pflegenden} In Bochum trafen sich 139 Pflegende aus allen Transplantationszentren Deutschlands, um neue Aspekte in der Transplantationspflege und -medizin vorzustellen und zu diskutieren. Die stetig ansteigende Teilnehmerzahl zeigt, wie groß das Interesse an diesem Thema bei den Pflegenden ist. Wie immer war das Symposium an die Jahrestagung der Deutschen Transplantationsgesellschaft gekoppelt. Der Tagungspräsident Prof. Richard Viebahn, Bochum, hob in seinem Grußwort hervor, dass von den Pflegesymposien bisher spürbare Inspirationen und Neuentwicklungen für die Transplantationsabteilungen ausgingen. Besonders würdigte er auch die Rolle der Krankenpflege bei der Betreuung hirntoter Organspender: Ohne die Anpassung des Berufsbildes und die individuelle Bereitschaft des

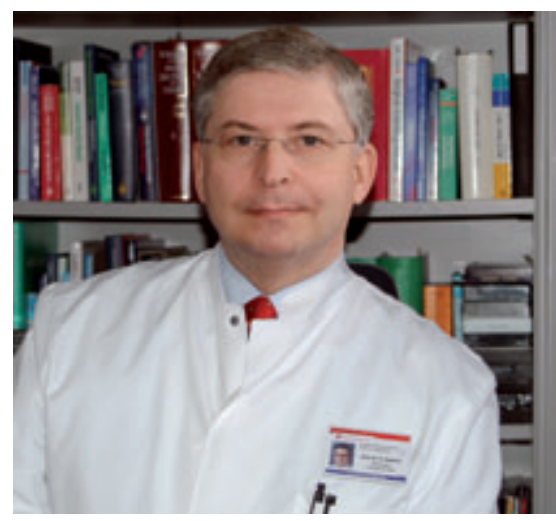

Prof. Richard Viehbahn.
Pflegepersonals auf Intensivstationen wäre die Organspende undenkbar.

Thomas Kissinger, Pflegedirektor des Knappschaftskrankenhauses Bochum, begrüßte uns mit den Worten: „In Zeiten der Veränderungen, die bestimmt sind durch Diskussionen zur Gesundheitsreform, Einsparungen in den Krankenhäusern und damit einhergehender Arbeitsverdichtung ist es umso wichtiger, sich den permanenten Veränderungsprozessen, auch in der Patientenversorgung, zu stellen und seine berufliche Kompetenz zu stärken durch den oft unterschätzten Aspekt der Fortbildung. Insofern leistet das 12. Pflegesymposium der AKTX einen Beitrag dazu, die Fachlichkeit und beruflichen Fähigkeiten der Pflegekräfte in den Transplantationszentren zu unterstützen."

\section{Patientenberatung und -schulung im Fokus}

Den Anfang machte Norbert Gittler-Hebestreit, Pflegewissenschaftler aus Jena, der eine Studie zur ambulanten Pflegeberatung nach Stammzelltransplanta- Norbert Gittler-Hebestreit. tionen vorstell-

te. Deren Aufgabe soll es sein, die Patienten und ihre Angehörigen mittel- und langfristig zu befähigen, bestehende und neu auftretende transplantationsbedingte Pflegeprobleme möglichst unabhängig von einer professionellen Unterstützung zu meistern. Ziel der Studie war es, ein strategisches und inhaltliches Konzept für eine ambulante Pflegeberatung nach einer Stammzelltransplantation zu entwickeln. Das entstandene Beratungskonzept wird jetzt am Universitätsklinikum in Jena umgesetzt.

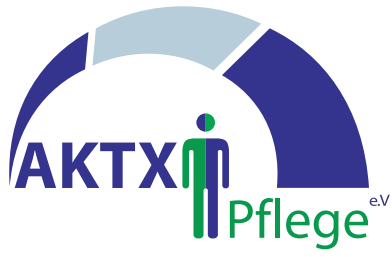

Ein immer wiederkehrendes Thema auf unseren Symposien ist die Patientenschulung. Ein Vortrag und 2 Poster beschäftigten sich mit dieser wichtigen Thematik. Der Vortrag von Dietmar Wiederhold, Nephrologisches Zentrum Niedersachsen, Hann. Münden, beinhaltete eine interessante Herangehensweise: Im Rahmen einer Studie wurden Patienten befragt, um Erkenntnisse aus deren subjektiver Perspektive zu erhalten. Bisher war nämlich unklar, wie Patienten ihre neue Situation kurz nach der Nierentransplantation erleben und welche Inhalte und Konsequenzen sich für eine nachfolgende Patientenschulung daraus ergeben. So lassen sich Verhaltensweisen und Reaktionen der Betroffenen besser verstehen, um somit schneller durch gezielte Schulungen professionell darauf reagieren zu können. Pflegende müssen von der ersten Stunde nach einer NTX adäquat auf die Patientenbedürfnisse eingehen können, um schwerwiegende Komplikationen (z.B. Abstoßungsreaktionen) möglichst zu verhindern. Daher kann es für Schwestern und Pfleger sehr hilfreich sein, subjektive Erlebnismuster der Patienten zu kennen und einordnen zu können.

\section{Das österreichische} Transplantationsgesetz

Stefan Hammer und Elisabeth Bierbaumer, AKH Wien, berichteten über die

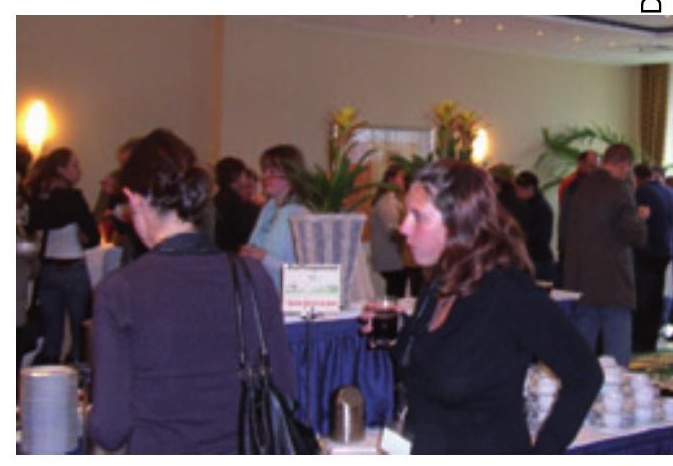

Teilnehmer des Pflegesymposiums. 


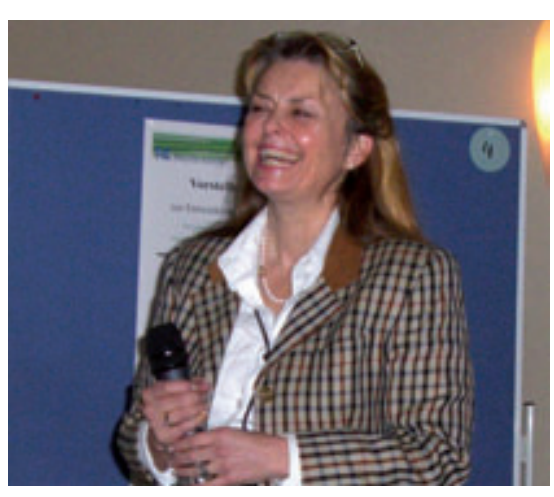

Dr. Gertrud Greif-Higer.

Pflege nach einer Transplantation nach „Wiener Art“. Ihre Erfahrungen mit dem österreichischen Transplantationsgesetz, welches die Widerspruchslösung bevorzugt, waren sehr interessant.

\section{Die psychosomatische Behandlung} ist sinnvoll und notwendig

Über die Wichtigkeit einer psychosomatischen Behandlung vor und nach einer Transplantation sprach Dr. Gertrud Greif-Higer, Psychologin von der Uniklinik Mainz. Durch den eklatanten Organmangel entstehen für viele Patienten lange Wartezeiten, die ihnen enorme körperliche und seelische Anstrengungen aufbürden. Viele verbringen Monate in stationärer Behandlung in einem Stadium zwischen Leben und Tod. Vor allem für die Pflegenden bedeutet dies oft eine große Belastung: Sie müssen die Patienten nicht nur behandeln, sondern ihnen auch eine darüber hinausgehende Stütze geben und oft auch die angespannte seelische Lage, die sich in Gereiztheiten äußern kann, aushalten. Auch werden viele Patienten in einem schlechten körperlichen Zustand transplantiert, der eine lange stationäre Behandlung erforderlich macht. Eine psychosomatische Behandlung scheint aus diesen vielschichtigen Gründen von äußerster Wichtigkeit, um gute Langzeitüberlebensraten zu erzielen trotz der oft widrigen Umstände.

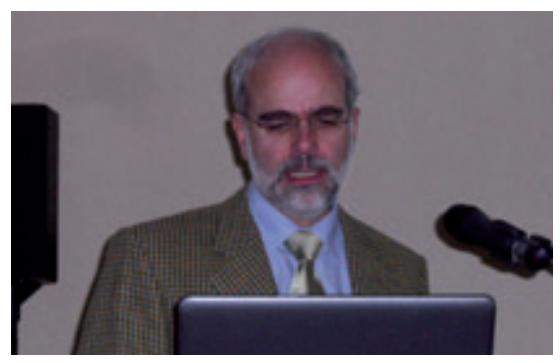

PD Roland Schulz-Röbbecke.
Immunsuppressiva sind seit jeher ein aktuelles Thema

Bei den vergangenen Symposien wurden in regelmäßigen Abständen Immunsuppressiva vorgestellt, so auch bei diesem Symposium: Dr. Wolfgang Arns stellte Anti-Thymozytenglobulin (ATG, Thymoglobulin ${ }^{\circledR}$ ) vor. Neben der Prävention und Behandlung von akuten Abstoßungsreaktionen hat ATG auch einen positiven Effekt auf die verzögerte Transplantatfunktion nach einer Nierentransplantation. Im Rahmen einer Induktionstherapie - also der Gabe vor der Transplantation - konnte die Rate der verzögerten Funktionsaufnahme halbiert werden.

Nach einer Organtransplantation stellen Infektionen weiterhin eine schwerwiegende Komplikation dar. Über infektionspräventive Maßnahmen nach einer Transplantation berichtete PD Roland Schulze-Röbbecke, Universitätsklinikum Düsseldorf. Für die Pflegenden bei ihrer täglichen Arbeit von besonderer Bedeutung sind die Erreger, die von exogen (d.h. von einer Quelle außerhalb des Organempfängers) in den Körper des Empfängers gelangen. Dies lässt sich durch Präventivmaßnahmen wie Händedesinfektion, Gebrauch von Einmalhandschuhen und gegebenenfalls dem Tragen eines Mund-NasenSchutzes verhindern.

\section{Tumor- und Herzerkrankungen bedrohen das Patientenwohl}

Einen sehr interessanten Vortrag hielt Dr. Claas Ulrich über Hauterkrankungen bei Organtransplantierten. Neu entwickelte und nebenwirkungsärmere Immunsuppressiva steigerten die durchschnittliche Überlebenszeit nach einer Transplantation deutlich. Die langzeitige Immunsuppression bedingt jedoch die unterschiedlichsten direkten und indirekten Therapiefolgen und Risiken.

Im Verlauf sind neben kardiovaskulären Risiken besonders Tumorerkrankungen die größte vitale Bedrohung für Organtransplantierte. Dabei stellen Hauttumoren mit 50\% den Hauptanteil der malignen Tumoren, die aber sehr gut behandelbar sind. Besonders gefährdet sind Transplantierte, die sich ungeschützt der Sonne aussetzen. Bereits bei der dermatologischen Untersuchung im Rahmen der Evaluation vor der Aufnahme auf die Warteliste sollte über UVSchutzmaßnahmen und regelmäßige
Selbstuntersuchungen informiert und aufgeklärt werden.

Aufklärungs- und Präventionsbroschüren, entwickelt für die besonderen Anforderungen und Fragen organtransplantierter Patienten sollten dem Patienten zusammen mit einer Probe hochwertiger (LSF 50) Sonnenschutzcreme bereits bei Aufnahme auf die Warteliste mitgegeben werden. Aber auch zu späteren Zeitpunkten nach Transplantationen hat die konsequente Anwendung eines hochwirksamen Sonnenschutzmittels eine große Bedeutung. Außerdem sollten die Patienten langärmelige Kleidung tragen. Bei der Schulung der Patienten nach einer Transplantation sollte das Pflegepersonal die Patienten dahingehend informieren und auch im Rahmen der Nachsorge sollten regelmäßige hautärztliche Untersuchungen erfolgen.

Elke Scholz, Herzzentrum Leipzig, stellte im nächsten Beitrag den Pflegestandard bei Herz- und Lungentransplantationen Ihres Hauses vor. Wie sie uns mitteilte, wird das Herzzentrum die Zusammenarbeit mit der Selbsthilfegruppe vor Ort sehr vielseitig gestalten. Von den Kollegen Robert Krawczyk, Agnes Dziuk und Peter Wessel, Bochum, hörten wir, wie ihre Erfahrungen mit Nierenlebendspenden bei ABO-inkompatiblen Spender-EmpfängerKonstellationen sind. Dieses Verfahren bei einer Nierenlebendspende wird zunehmend in mehreren Zentren angewendet.

\section{Auch die Poster trugen zum Erfolg des Symposiums bei}

Zum Pflegesymposium des AKTX gehört seit einigen Jahren wieder eine Posterausstellung. In diesem Jahr wurden 7 Poster vorgestellt. Die Vielfalt der Posterthemen bestätigt abermals das breite Spektrum der beruflichen Kompetenz der Pflegenden im Bereich Transplantation. Die Vorträge und Poster sind nachzulesen unter: www.aktxpflege.de

Der Tag war für alle Teilnehmer sicher ein Zugewinn an Information und Wissen. Insofern hat das 12. Pflegesymposium des AKTX wieder einen Beitrag dazu geleistet, die Kompetenz und die beruflichen Fähigkeiten der Pflegekräfte in den Transplantationszentren zu unterstützen. Dies will der Arbeitskreises Transplantationspflege auch im nächsten Jahr in Berlin mit dem 13. Pflegesymposium in gleicher Qualität fortsetzen.

Esther Ziemann, Pressewart AKTX-Pflege e.V. 